\title{
Budesonide Use and Hospitalization Rate in Crohn's Disease: Results From a Cohort at a Tertiary Care IBD Referral Center
}

\author{
Jordan Orr ${ }^{\mathrm{a}, \mathrm{d}}$, Krishna V. R. Venkata ${ }^{\mathrm{b}}$, Steven Younga ${ }^{\mathrm{a}}$, Fenglong Xie ${ }^{\mathrm{c}}$, Talha A. Malik ${ }^{\mathrm{c}}$
}

\begin{abstract}
Background: Budesonide is generally not used for periods $>90$ days in Crohn's disease (CD). We sought to study the association between cumulative outpatient budesonide use in days and hospitalization rate in $C D$ patients seen at our institution.

Methods: Using a retrospective cohort study design, we selected CD patients $>19$ years old and followed for at least 1 year. Days of outpatient budesonide use were calculated by reviewing outpatient clinic notes. Treatment groups included patients who were not given budesonide, received budesonide from 1 to 90 days, and received budesonide $>90$ days. We performed univariate analyses and developed generalized Poisson regression models for rate data to estimate incidence rate ratios (IRRs) and 95\% confidence intervals (95\% CIs) for CD-related hospitalization.
\end{abstract}

Results: Of $767 \mathrm{CD}$ patients, 664 did not receive budesonide, 45 received budesonide from 1 to 90 days, and 58 received budesonide for $>90$ days. Incidence rates of hospitalization in patients who received no budesonide vs. 1 - 90 days of budesonide vs. $>90$ days of budesonide were 31,26 , and 19 per 100 person-years, respectively. Adjusted models demonstrated that receiving outpatient budesonide from 1 to 90 days and for $>90$ days was associated with a lower likelihood of being admitted for a CD exacerbation ( 1 - 90 days: IRR 0.85 ; $95 \% \mathrm{CI}$ 0.65 - 1.10; > 90 days: IRR $0.71 ; 95 \%$ CI $0.56-0.91)$.

Conclusions: Outpatient budesonide use appears to be associated with a lower likelihood of a CD-related hospitalization, notably when used for $>90$ days. This association needs to be further assessed before recommending this agent for routine use for $>90$ days.

Manuscript accepted for publication July 04, 2016

aDepartment of Internal Medicine, Tinsley Harrison Internal Medicine Residency Program, University of Alabama at Birmingham, 1720 2nd Ave South, BDB 327, Birmingham, AL 35294, USA

${ }^{b}$ Department of Medicine, University of Alabama at Birmingham, Montgomery Internal Medicine Residency Program, 2055 E. South Blvd, Suite 200, Montgomery, AL 36116, USA

'Division of Gastroenterology \& Hepatology, University of Alabama at Birmingham, 1808 7th Ave South, BDB 391, Birmingham, AL 35294, USA

${ }^{\mathrm{d} C}$ Corresponding Author: Jordan Orr, Department of Internal Medicine, The University of Alabama at Birmingham, 1720 2nd Ave South, BDB 327, Birmingham, AL 35294, USA. Email: jordanorr@uabmc.edu

doi: http://dx.doi.org/10.14740/jocmr2630w
Keywords: Crohn's disease; Budesonide; Hospitalization rate; Outcomes

\section{Introduction}

Crohn's disease (CD) is a disease of complex inflammatory pathophysiology resulting in significant morbidity and mortality and is characterized as a chronic relapsing disease leading to hospitalization and often surgery. Approximately 780,000 people in the United States are afflicted with this disease with an increasing trend of hospitalization and health care costs despite advances in medical therapy $[1,2]$. Conventional corticosteroids are a proven tool for inhibiting the cytokine and chemokine cascade that results in the chronic dysregulated proinflammatory response that defines CD [3]. Since their therapeutic introduction in the 1940s, corticosteroids have been a mainstay in the treatment of inflammatory bowel disease (IBD) and produce a potent anti-inflammatory effect $[4,5]$. Unfortunately, the use of corticosteroids is not benign and their long-standing use is limited due to the potential for severe adverse effects. New formulations of corticosteroids have been designed and implemented in an attempt to limit the systemic adverse effects of these medications. Budesonide is such an agent that is a targeted synthetic corticosteroid that has high local efficacy in the gastrointestinal (GI) tract and minimal systemic adverse effects. Budesonide undergoes extensive first-pass metabolism whereby the liver metabolizes $80-90 \%$ of the parent compound resulting in significantly less bioavailability and, thus, less systemic adverse effects [6]. Current guidelines and traditional convention do not support the use of budesonide for maintenance therapy greater than 3 months. The purpose of this study was to evaluate the association between cumulative outpatient budesonide use in terms of days and hospitalization rate in CD patients seen at our institution.

\section{Materials and Methods}

\section{Study design, patient population, and selection criteria}

We conducted a retrospective cohort study following approval by the University of Alabama's Office of Institutional Review 
Table 1. Demographic and Clinical Characteristics by Budesonide Use

\begin{tabular}{|c|c|c|c|}
\hline Characteristic & No budesonide use $(n=664)$ & Budesonide use 1 - 90 days $(n=45)$ & Budesonide use $>90$ days $(n=58)$ \\
\hline Age in years, mean (SD) & $32.3(13.6)$ & $32.9(14.0)$ & $29.9(11.5)$ \\
\hline DoD (SD) & $12.5(10.5)$ & $15.7(15.5)$ & $17.6(9.4)$ \\
\hline \multicolumn{4}{|l|}{ Race, $\%$} \\
\hline Caucasian & 74.4 & 77.8 & 75.9 \\
\hline \multicolumn{4}{|l|}{ Sex, \% } \\
\hline Female & 62.5 & 71.1 & 67.2 \\
\hline Metabolic syndrome, $\%$ & 4.2 & 11.1 & 1.7 \\
\hline Obesity, $\%$ & 19.6 & 17.8 & 17.2 \\
\hline Methotrexate use, $\%$ & 10.5 & 8.9 & 13.8 \\
\hline
\end{tabular}

DoD: duration of disease (years); DoO: duration of observation (years); SD: standard deviation.

Board (IRB). We analyzed data from $767 \mathrm{CD}$ patients seen at our tertiary care IBD center from 2000 to 2012 and followed for at least 1 year. Selection criteria included age $>19$ years old, duration of observation $\geq 1$ year, duration of disease of $\geq$ 1 year, and sufficient electronic medical record (EMR) documentation. Sufficient documentation meant that the EMR had at least one physician note that contained medical history and was dated from within the first year of observation. Other variables controlled for included duration of disease, race, sex, obesity, diagnosis of metabolic syndrome, and usage of biologic and traditional immunomodulator (azathioprine, 6-mercaptopurine, and methotrexate) therapy.

\section{Data collection and variable definitions}

Data were collected by retrospective chart review per EMR documentation. Days of outpatient budesonide use were calculated by reviewing documented budesonide use in clinic notes of each patient. Days of inpatient budesonide use were not included. Data collected at the time of first observation included age, race, sex, duration of $\mathrm{CD}$, and total number of days of documented steroid use. A CD-related hospitalization was defined as any hospital admission for a complication of CD, including infections, fistula, strictures, abscess, or exacerbations. Patients were considered to be on an immune modulator if they were on any biological agent or traditional immunomodulator for at least 4 weeks during the period of observation. The period of observation was defined as the time in years between the first and the last documented encounter at our tertiary care IBD center during the years 2000 through 2012. Obesity was defined by using standard body mass index (BMI) formula. Patients were considered to have metabolic syndrome if they had the diagnosis of at least three of the following at the time of beginning of observation: diagnosis of hypertension, diabetes mellitus, hypertriglyceridemia, low HDL levels, or obesity (ATP III criteria).

\section{Statistical analysis}

After calculating summary statistics, we performed univariate analyses to examine the incidence rate for CD-related hospitalizations among CD patients based on outpatient budesonide use. Patients were divided into three categories based on outpatient budesonide use: those who were not given budesonide, those who received budesonide from 1 to 90 days, and those who received budesonide for $>90$ days. We built generalized Poisson regression models for rate data to estimate partially adjusted (for age, sex, race, and duration of disease) and fully adjusted (additionally for biologic and traditional immunomodulator use, diagnosis of metabolic syndrome, and obesity) incidence rate ratios (IRRs) of hospitalization among CD patients with no budesonide use, those who received budesonide for 1 - 90 days, and those who received budesonide for $>90$ days during the entire follow-up period. All analyses were conducted using SAS 9.4 (SAS Institute, Cary, NC).

\section{Results}

From our cohort of $767 \mathrm{CD}$ patients seen at our institution, 664 did not receive budesonide, 45 received budesonide for 1 - 90 days, and 58 received budesonide for $>90$ days (Table 1). Incidence rates for CD-related hospitalizations in patients who received budesonide for 1 - 90 days and for $>90$ days were 25.86 and 19.47 per 100 person-years, respectively, compared to 30.68 per 100 person-years in patients who did not receive 
Table 2. Crude, Partially Adjusted, and Fully Adjusted Incidence Rate Ratios for CD-Related Hospitalizations

\begin{tabular}{llllll}
\hline \multirow{2}{*}{ Budesonide use } & $\begin{array}{l}\text { CD-related number of } \\
\text { hospitalizations/per } \\
\text { 100-person years }\end{array}$ & $\begin{array}{l}\text { Hospitalization } \\
\text { rate (95\% CI)* }\end{array}$ & Model 1 & Model 2 & Model 3 \\
\hline No budesonide use & $924 / 3,012$ & $30.68(28.76-32.72)$ & 1 (reference) & 1 (reference) & 1 (reference) \\
Budesonide use 1 - 90 days & $68 / 263$ & $25.86(20.39-32.79)$ & $0.84(0.66-1.08)$ & $0.97(0.76-1.24)$ & $0.85(0.65-1.10)$ \\
Budesonide use $>$ 90 days & $89 / 457$ & $19.47(15.82-23.97)$ & $0.63(0.51-0.79)$ & $0.67(0.54-0.83)$ & $0.71(0.56-0.91)$ \\
\hline
\end{tabular}

*Per 100 person-years. Model 1 is unadjusted. Model 2 is partially adjusted for age, sex, race, and duration of disease. Model 3 is fully adjusted for age, sex, race, duration of disease, biologic and traditional immune modulators, diagnosis of metabolic syndrome, and obesity. Cl: confidence interval; RR: rate ratio.

budesonide (Table 2). Results from unadjusted generalized Poisson regression models demonstrated a $16 \%$ lower likelihood for a CD-related hospitalization in patients who received budesonide for 1 - 90 days when compared to patients who did not receive budesonide, but these results did not display statistical significance (IRR $0.84 ; 95 \%$ CI 0.66 - 1.08). The partially and fully adjusted models also confirm this clinically significant association but did not confer statistical significance (IRR 0.97; 95\% CI 0.76 - 1.24 and IRR 0.85; 95\% CI $0.65-1.10$, respectively). Results from unadjusted generalized Poisson regression models demonstrated a 37\% lower likelihood for a CD-related hospitalization in patients who received budesonide for $>90$ days when compared to patients who did not receive budesonide, both clinically and statistically significant (IRR $0.63 ; 95 \%$ CI $0.51-0.79$ ). The partially and fully adjusted model confirmed this clinically and statistically significant association (IRR 0.67; 95\% CI 0.56 - 0.91 and IRR $0.71 ; 95 \%$ CI 0.56 - 0.91, respectively). Detailed results are shown in Table 2.

\section{Discussion}

Cumulative outpatient budesonide use appears to be associated with a lower likelihood of a CD-related hospitalization. We demonstrated that CD patients who received budesonide for 1 - 90 days as an outpatient had a 15\% lower likelihood of being admitted for a CD exacerbation (IRR 0.85; 95\% CI 0.65 - 1.10, fully adjusted model) and, further, CD patients receiving budesonide for $>90$ days had an even lower likelihood of being hospitalized for a CD exacerbation (IRR 0.71; 95\% CI $0.56-0.91$, fully adjust model). These findings support the notion that budesonide use may be feasible for a period greater than 3 months.

Studies have proven the efficacy of budesonide for induction of remission in CD. Greenberg et al compared the efficacy of budesonide to placebo in a large multicenter trial with 258 patients and found that after 8 weeks of treatment, patients receiving budesonide achieved greater rates of remission as compared to placebo [7]. This trial confirmed the efficacy of budesonide in the induction of remission of CD and established the $9 \mathrm{mg}$ daily dose as the most effective dose $(51 \%$ remission in patients receiving $9 \mathrm{mg}$ budesonide daily compared to $20 \%$ remission in patients receiving placebo, $\mathrm{P}<0.001)[5,7]$. A Cochran review including 14 studies (1,805 patients) compar- ing budesonide to conventional corticosteroids, placebo, and mesalamine found that after 8 weeks of treatment, $9 \mathrm{mg}$ budesonide was significantly more effective than placebo $(47 \%$ remission with budesonide vs. $22 \%$ remission with placebo, RR 1.93; 95\% CI 1.37 - 2.73) and mesalamine (68\% remission with budesonide vs. $42 \%$ remission with mesalamine, RR $1.63 ; 95 \%$ CI 1.23 - 2.16) for induction of remission, however less effective than conventional corticosteroids for induction of remission (52\% remission with budesonide vs. $61 \%$ remission with conventional steroids, RR 0.85 ; 95\% CI 0.75 - 0.97 ) [8]. These findings confirm that budesonide has proven to be an effective agent for induction to remission in CD.

The efficacy of long-term budesonide use in CD is less defined and is generally felt not effective for the maintenance of remission in CD. Greenburg et al found no difference in relapse rates at the 1-year follow-up when comparing budesonide to placebo [9]. Further, a 2009 Cochran review found budesonide to be no better than placebo at maintaining remission at 12 months [10]. A more recent Cochran review of eight studies compared use of budesonide ( $6 \mathrm{mg}$ daily) to placebo for maintenance of remission and concluded budesonide was no more effective than placebo for maintenance of remission at 3 months (RR 1.25; 95\% CI 1.00 - 1.58), 6 months (RR 1.15; $95 \%$ CI 0.95 - 1.39), or 12 months (RR 1.13; 95\% CI 0.94 - 1.35) [11]. As such, the American Gastroenterological Association guidelines recommend budesonide therapy for 8 weeks for induction of remission and for an additional 3 months of maintenance treatment in a single year [12].

There is some benefit, though, for budesonide use in maintenance of remission in CD. Patients treated with daily budesonide for 1 year display a lower Crohn's disease activity index (CDAI) score, a grading tool for CD severity, and also have a longer time to relapse $[13,14]$. This prolonged time to relapse associated with budesonide was demonstrated in a randomized, double blind, multicenter trial study by Lofberg et al. This study distributed 176 patients with active CD who had achieved remission (CDAI index score $\leq 150$ ) into treatment arms receiving budesonide $6 \mathrm{mg}$ daily, budesonide 3 $\mathrm{mg}$ daily, or placebo and after 3 months found relapse rates of $19 \%, 45 \%$, and $44 \%$ for the $6 \mathrm{mg}$ daily, $3 \mathrm{mg}$ daily, and placebo groups, respectively. Results were less statistically significant when extended to 12 months; however, the median time to relapse was 258 days in the $6 \mathrm{mg}$ group, 139 days in the $3 \mathrm{mg}$ group, and 92 days in the placebo group $(\mathrm{P}=0.021)$ [15]. Results from our study show a similar effect of cumula- 
tive budesonide use, in that, patients receiving budesonide for an extended period of time, specifically $>90$ days, decrease the risk of being hospitalized for a CD exacerbation.

Because of the retrospective nature of this study, we were not truly able to measure adverse effects (AEs) associated with budesonide use or other reasons for hospitalization. Perhaps most concerning with cumulative use of budesonide is the possibility for AEs. Systemic corticosteroids, such as prednisone or prednisolone, are known for their significant systemic AEs. The combination of localized delivery, high topical glucocorticoid receptor activity, and considerable firstpass metabolism in the liver makes budesonide a much safer choice in terms of systemic AEs [16]. Studies showing significant increased median time to clinical relapse using budesonide for 1 year compared to placebo also demonstrated a safety profile similar to placebo [17]. Thus, clinicians should not be overly concerned regarding the risk of AEs from using budesonide for $\geq 90$ days.

Lichentein et al performed a pooled safety analysis reviewing five 1-year, double blind, placebo-controlled trials (417 patients) to evaluate the safety profile of budesonide for mild to moderate CD. Of all potential AEs, the highest incidence rates of AEs were gastrointestinal (nausea, dyspepsia, vomiting, epigastric pain, reflux, and ulcerations) and endocrine system-related, caused by a higher overall occurrence of cutaneous corticosteroid symptoms (moon face, acne, swelling, easy bruising, hirsutism, buffalo hump, and striae). The clinically important or serious AEs such as sepsis, cataracts, adrenal insufficiency, fractures, or thrombosis and arterial embolism were rare and rated similar between treatment groups [16]. Kuenzig et al in a Cochran review concluded that AEs were not more common in patients treated with budesonide compared to placebo at both 6 and 12 months and the AEs observed in this review were relatively minor and did not result in increased rates of study withdrawal [11].

The results of this study were found using patients who received care from our tertiary care IBD center and generally represent $C D$ patients with severe disease. Variables not controlled for in this study include tobacco use, other steroid use (conventional corticosteroids or rectal/topical steroids), clinical disease and severity (CDAI), daily budesonide dose, and maintenance of remission. It is conceivable that $\mathrm{CD}$ patients with worse clinical disease (higher CDAI score) required more aggressive therapy with conventional corticosteroids and did not receive budesonide and likely contributed to more CDrelated hospitalizations in the no budesonide arm. Our cohort likely included patients with both mild and severe disease, therefore, it is felt these results can be applied to all CD patients; however, it would be helpful to adjust for clinical disease and severity in the future.

This study was intended to examine the relationship between cumulative budesonide use and rates of CD-related hospitalizations. Our results suggest that CD patients receiving budesonide for $>90$ days have significantly less rates of hospitalization. This association warrants further investigation to better understand if budesonide can effectively and safely be administered for $>90$ days. Studies evaluating budesonide use in the future should assess other outcome parameters associated with increased budesonide use and adjust outcomes for clinical disease and severity, budesonide dose, and other steroid use to further elucidate the role of budesonide in CD. Budesonide is a powerful tool in the treatment of CD and the findings from our study may contribute to guideline adjustment for the recommended use of budesonide in $\mathrm{CD}$ in the future.

\section{Disclosure}

There are no potential conflicts of interest to disclose in the presentation of this publication for all authors involved. Also, there are no funding sources or any other types of assistance for this publication to disclose.

\section{Author Contributions}

Jordan Orr, Krishna Venkata, Steven Young, and Talha Malik conceptualized the study hypothesis, design, and methodology. Jordan Orr, Krishna Venkata, and Steven Young collected data for study. Fenglong Xie performed the statistical analysis. Jordan Orr and Krishna Venkata drafted the manuscript. Talha Malik contributed in critical revision of the manuscript for important intellectual content, expert opinion, and study supervision. All authors approved the final draft submitted. Jordan Orr is the author guarantor.

\section{References}

1. Bewtra M, Su C, Lewis JD. Trends in hospitalization rates for inflammatory bowel disease in the United States. Clin Gastroenterol Hepatol. 2007;5(5):597-601.

2. Crohn's \& Colitis Foundation of America. The Facts About Inflammatory Bowel Diseases. New York, NY: Crohn's \& Colitis Foundation of America; 2014.

3. Lasher BA. Crohn's Disease. Disease Management. Lyndhurst, OH: The Cleveland Clinic Foundation; 2013.

4. Prantera C, Marconi S. Glucocorticosteroids in the treatment of inflammatory bowel disease and approaches to minimizing systemic activity. Therap Adv Gastroenterol. 2013;6(2):137-156.

5. Yang YX, Lichtenstein GR. Corticosteroids in Crohn's disease. Am J Gastroenterol. 2002;97(4):803-823.

6. Nunes T, Barreiro-de Acosta M, Marin-Jimenez I, Nos P, Sans M. Oral locally active steroids in inflammatory bowel disease. J Crohns Colitis. 2013;7(3):183-191.

7. Greenberg GR, Feagan BG, Martin F, Sutherland LR, Thomson AB, Williams CN, Nilsson LG, et al. Oral budesonide for active Crohn's disease. Canadian Inflammatory Bowel Disease Study Group. N Engl J Med. 1994;331(13):836-841.

8. Rezaie A, Kuenzig ME, Benchimol EI, Griffiths AM, Otley AR, Steinhart AH, Kaplan GG, et al. Budesonide for induction of remission in Crohn's disease. Cochrane Database Syst Rev. 2015;6:CD000296.

9. Greenberg GR, Feagan BG, Martin F, Sutherland LR, 
Thomson AB, Williams CN, Nilsson LG, et al. Oral budesonide as maintenance treatment for Crohn's disease: a placebo-controlled, dose-ranging study. Canadian Inflammatory Bowel Disease Study Group. Gastroenterology. 1996;110(1):45-51.

10. Benchimol EI, Seow CH, Otley AR, Steinhart AH. Budesonide for maintenance of remission in Crohn's disease. Cochrane Database Syst Rev. 2009;1:CD002913.

11. Kuenzig ME, Rezaie A, Seow CH, Otley AR, Steinhart AH, Griffiths AM, Kaplan GG, et al. Budesonide for maintenance of remission in Crohn's disease. Cochrane Database Syst Rev. 2014;8:CD002913.

12. Lichtenstein GR, Abreu MT, Cohen R, Tremaine W. American Gastroenterological Association Institute medical position statement on corticosteroids, immunomodulators, and infliximab in inflammatory bowel disease. Gastroenterology. 2006;130(3):935-939.

13. Best WR, Becktel JM, Singleton JW, Kern F, Jr. Development of a Crohn's disease activity index. National Cooperative Crohn's Disease Study. Gastroenterology.
1976;70(3):439-444.

14. Hanauer S, Sandborn WJ, Persson A, Persson T. Budesonide as maintenance treatment in Crohn's disease: a placebo-controlled trial. Aliment Pharmacol Ther. 2005;21(4):363-371.

15. Lofberg R, Rutgeerts P, Malchow H, Lamers C, Danielsson A, Olaison G, Jewell D, et al. Budesonide prolongs time to relapse in ileal and ileocaecal Crohn's disease. A placebo controlled one year study. Gut. 1996;39(1):8286.

16. Lichtenstein GR, Bengtsson B, Hapten-White L, Rutgeerts P. Oral budesonide for maintenance of remission of Crohn's disease: a pooled safety analysis. Aliment Pharmacol Ther. 2009;29(6):643-653.

17. Sandborn WJ, Lofberg R, Feagan BG, Hanauer SB, Campieri M, Greenberg GR. Budesonide for maintenance of remission in patients with Crohn's disease in medically induced remission: a predetermined pooled analysis of four randomized, double-blind, placebo-controlled trials. Am J Gastroenterol. 2005;100(8):1780-1787. 